\title{
Adjuvant Chemotherapy of Gemcitabine plus Carboplatin versus Paclitaxel plus Carboplatin in Patients with Resected Non-Small Cell Lung Cancer
}

\author{
Takanori Ayabe*, Masaki Tomita, Kunihide Nakamura \\ Department of Surgery II, Faculty of Medicine, University of Miyazaki, Miyazaki, Japan. \\ Email: *tayabe@med.miyazaki-u.ac.jp
}

Received June $13^{\text {th }}, 2013$; revised July $15^{\text {th }}, 2013$; accepted July $23^{\text {rd }}, 2013$

Copyright (C) 2013 Takanori Ayabe et al. This is an open access article distributed under the Creative Commons Attribution License, which permits unrestricted use, distribution, and reproduction in any medium, provided the original work is properly cited.

\begin{abstract}
Background: This retrospective study was to evaluate the efficacy and toxicity of gemcitabine plus carboplatin (GC regimen) and paclitaxel plus carboplatin (PC regimen) combination chemotherapy administered as an adjuvant therapy after complete resection of non-small cell lung cancer. Methods: Forty-four patients (GC regimen, $\mathrm{n}=29$; PC regimen, $\mathrm{n}=15$ ) received gemcitabine at a dose of $1000 \mathrm{mg} / \mathrm{m}^{2}$ on days 1 and 8 , and carboplatin with the target dose of area under the curve (AUC) of 4 on day 8 every 28 days and paclitaxel at a dose of $70 \mathrm{mg} / \mathrm{m}^{2}$ on days 1,8 and 15 , and carboplatin with the target dose of AUC of 5 on day 1 every 28 days. Results: A total of 130 cycles of the treatment were administered (averaged, 3.1 in GC arm and 2.7 cycles in PC arm). Forty-three patients (97.7\%) completed the scheduled cycles. One patient $(2.3 \%)$ was discontinued due to grade 4 pneumonia. The dose was reduced in 2 patients $(4.5 \%)$ due to grade 4 thrombocytopenia. Grade 3/4 neutropenia was significantly observed in the PC group (GC: $12 / 29,41.4 \%$; PC: $11 / 15,73.3 \%, p=0.0443)$. The nonhematological toxicities were mild. Grade $1 / 2$ alanine aminotransferase and aspartate aminotransferase in the GC group was significantly observed higher compared to those of the PC group (GC: 20/29, $69.0 \%$; PC: $4 / 15,26.7 \%, p=0.0076)$. Grade $1 / 2$ alopecia was significantly observed in the PC group (GC: $0 / 25,0.0 \%$; PC: $13 / 15,86.7 \%, \mathrm{p}<0.0001)$. There was no treatment-related death. The median survival time (MST) of the entire GC group was 784 days, the 3-year overall survival (OS) was $75.9 \%$, and 3-year recurrence-free survival (RFS) was $65.5 \%$. The MST of the entire PC group was 963 days, the 3-year OS was $80.0 \%$, and the 3-year RFS was $60.0 \%$. Conclusion: These results demonstrate that the GC and PC combination chemotherapies are efficacious and feasible regimens, which should be considered as one of the standard therapies for adjuvant therapy.
\end{abstract}

Keywords: Non-Small Cell Lung Cancer; Gemcitabine; Paclitaxel; Carboplatin; Combination Chemotherapy; Adjuvant Therapy

\section{Introduction}

Non-small cell lung cancer (NSCLC) is currently the leading cause of death related to cancer $[1,2]$. The most effective treatment for the early stages (IA-IIIA) NSCLC is surgical resection. However, up to $60 \%$ of patients with IB to IIIA NSCLC relapse after surgery and die [2,3]. Adjuvant therapy for early-stage NSCLC has been the focus of much study in the hope of reducing the relapse risk and improving survival from the $40 \%$ to $60 \%$ achieved with surgery alone [2]. The presence of micrometastatic disease at the time of resection is the most likely cause of recurrence occurring even after complete resection of all

${ }^{*}$ Corresponding author. the macroscopically recognizable lesions. If micrometastases are indeed responsible for the disease recurrence, adjuvant chemotherapy would be a rational treatment, and this hypothesis has led to the attempt to reduce the risk of relapse and death from lung cancer by giving adjuvant chemotherapy to patients with complete surgical resection [4].

More recent randomized trials of adjuvant cisplatinbased chemotherapy have shown only marginally better compliance despite considerable improvements in the supportive care medications available over the past decade. North American [5], Japanese [6], and European [7-9] intergroup trials reported that only $58 \%-69 \%$ of patients received all of the planned cycles of chemotherapy.

A meta-analysis of small randomized trials of patients 
with early stage NSCLC in the preceeding 30 years was performed in 1995 [4]. This analysis revealed a 5\% survival advantage at 5 years for patients with surgically resected early stage NSCLC treated with cisplatin-based chemotherapy compared to those patients only followed up after resection.

It is not yet clear whether platinum-based chemotherapy is feasible or available in an adjuvant setting for Japanese patients. Adjuvant chemotherapy is the standard of therapy for some patients with stage I, II, and III breast cancers. The therapeutic efficacy of adjuvant chemotherapy following surgical resection of early stage NSCLC has been less clear. In clinical practice, we conducted trials to assess the efficacy and safety of two regimens in Japanese patients with NSCLC in a single institution. This retrospective study was to evaluate the efficacy and toxicity of gemcitabine plus carboplatin (GC regimen) and paclitaxel plus carboplatin (PC regimen) combination chemotherapy administered as an adjuvant therapy after complete resection of non-small cell lung cancer.

\section{Patients and Methods}

\subsection{Patients}

From December 2004 to July 2009, a total of 44 patients who had undergone surgical resection received adjuvant chemotherapy of the gemcitabine plus carboplatin or paclitaxel plus carboplatin doublet combinations. The characteristics of the 44 patients entered in this study are summarized in Tables $\mathbf{1}$ and 2. TNM classification is based on the Union for International Cancer Control (UICC) [10]. The histological analysis of the tumor was based on the World Health Organization classification for cell types [11]. Patients with histologically documented NSCLC and pathologically staged were eligible to receive adjuvant chemotherapy after complete resection of the primary tumor and mediastinal lymph nodes in our department.

\subsection{Each Patient Had to Meet the Following Eligibility Criteria}

Pathological stage from IB to IV diagnosed with complete resection, Eastern Cooperative Oncology Group Performance Status of 0,1 or 2, adequate bone marrow function (total leukocyte count $\geq 4.0 \times 10^{9} / 1$, hemoglobin concentration $\geq 10.0 \mathrm{~g} / \mathrm{dl}$, platelet count $\left.\geq 100 \times 10^{9} / 1\right)$, adequate liver and renal function (serum transaminase $\leq 2$ times normal value; serum creatinine $\leq 1.5$ times normal value), partial pressure of arterial oxygen $\left(\mathrm{paO}_{2}\right) \geq 60$ torr,

Table 1. Patient characteristics.

\begin{tabular}{|c|c|c|c|c|c|c|c|}
\hline & & \multicolumn{2}{|c|}{ GEM + CBDCA group $(n=29)$} & \multicolumn{2}{|c|}{ PTX + CBDCA group $(n=15)$} & \multicolumn{2}{|c|}{ Overall $(n=44)$} \\
\hline & & No. of patients & $(\%)$ & No. of patients & $(\%)$ & No. of patients & $(\%)$ \\
\hline \multirow{2}{*}{ Age (years) } & Mean $\pm \mathrm{SD}$ & $64.0 \pm 9.2$ & & $61.2 \pm 12.5$ & & $62.8 \pm 10.6$ & \\
\hline & range & $45-78$ & & $35-77$ & & $35-78$ & \\
\hline \multirow{2}{*}{ Gender } & Male & 18 & 62.1 & 13 & 86.7 & 31 & 70.5 \\
\hline & Female & 11 & 37.9 & 2 & 13.3 & 13 & 29.5 \\
\hline \multirow{2}{*}{ ECOG-PS } & 0 & 27 & 93.1 & 14 & 93.3 & 41 & 93.2 \\
\hline & 1 & 2 & 6.9 & 1 & 6.7 & 3 & 6.8 \\
\hline \multirow{2}{*}{ Surgery } & Lobectomy & 27 & 93.1 & 14 & 93.3 & 41 & 93.2 \\
\hline & Pneumonectomy & 2 & 6.9 & 1 & 6.7 & 3 & 6.8 \\
\hline \multirow{3}{*}{ Histology } & Adenocarcinoma & 26 & 89.7 & 13 & 86.7 & 39 & 88.6 \\
\hline & Squamous cell carcinoma & 2 & 6.9 & 1 & 6.7 & 3 & 6.8 \\
\hline & Adenosquamous cell carcinoma & 1 & 3.4 & 1 & 6.7 & 2 & 4.6 \\
\hline \multirow{6}{*}{ Clinical stage } & IB & 1 & 3.4 & 1 & 6.7 & 2 & 4.6 \\
\hline & IIA & 5 & 17.2 & 4 & 26.7 & 9 & 20.4 \\
\hline & IIB & 4 & 13.8 & 1 & 6.7 & 5 & 11.4 \\
\hline & IIIA & 14 & 48.3 & 8 & 53.3 & 22 & 50 \\
\hline & IIIB & 2 & 6.9 & 1 & 6.7 & 3 & 6.8 \\
\hline & IV & 3 & 10.3 & & & 3 & 6.8 \\
\hline
\end{tabular}

ECOG-PS: Eastern Cooperative Oncology Group Performance Status. 
Table 2. Treatment background.

\begin{tabular}{cccc}
\hline & & GEM + CBDCA group $(\mathrm{n}=29)$ & PTX + CBDCA group $(\mathrm{n}=15)$ \\
\hline Planned cycles & $1 / 2 / 3 / 4 / 5 / 6$ cycles & $0 / 11 / 5 / 13 / 0 / 0$ & $0 / 9 / 0 / 5 / 0 / 1$ \\
Received cycles & $1 / 2 / 3 / 4 / 5 / 6$ cycles & $0 / 11 / 5 / 13 / 0 / 0$ & $1 / 9 / 0 / 4 / 0 / 1$ \\
& Total cycles & 89 & 41 \\
& mean & 3.1 & 2.7 \\
Compliance & Completed & $29(100.0 \%)$ & $14(93.3 \%)$ \\
& Discontinued & $0(0.0 \%)$ & $1(6.7 \%)$ \\
Dose reduction & Delayed & $1(2.2 \%)$ & $0(0.7 \%)$ \\
\hline
\end{tabular}

*Grade 4 pneumonia.

past history of severe allergic reaction to drugs, interstitial pneumonia identified by computed-tomography of chest, cirrhosis, or other serious complications, such as uncontrolled angina pectoris, myocardial infarction within 3 months, heart failure, uncontrolled diabetes mellitus or hypertension, and uncontrolled massive pleural effusion or ascites, no postoperative complications, able to undergo first course treatment in an inpatients setting within 4 to 8 weeks after surgery, and written informed consent. All patients provided written informed consent before the treatment.

\subsection{Treatment Schedule}

All patients received one of the two treatment groups by attending doctors' direction and/or patients' favorable selection depending on their toxicities. The body surface area was calculated using the DuBois equation. Carboplatin dosage calculation was based on glomerular filtration rate according to the Calvert formula [12], and evaluated with the Cockcroft-Gault equation [13]. The administration of carboplatin dosage was adjusted prior to each cycle through re-determination of the glomerular filtration rate.

\subsubsection{Gemcitabine plus Carboplatin Regimen (GC Group)}

Gemcitabine (Gemzar, Eli Lilly Japan K.K., Kobe, Japan) was adminstered at a dose of $1000 \mathrm{mg} / \mathrm{m}^{2}$ on days 1 and 8, and carboplatin (Paraplatin, Bristol-Myers K.K., Tokyo, Japan) with the target dose of area under the curve (AUC) of 4 on day 8 every 28 days. Premedication was intravenously performed with drip infusion of $100 \mathrm{ml}$ of isotonic sodium chloride solution containing of $8 \mathrm{mg}$ dexamethasone sodium phosphate and $3 \mathrm{mg}$ of granisetron hydrochloride. An infusion pump was used to ensure the exact infusion time. On days 1 and 8 , the intravenous administration of $1000 \mathrm{mg} / \mathrm{m}^{2}$ gemcitabine mixed in 100 $\mathrm{ml}$ of isotonic sodium chloride solution was performed with drip infusion for 30 minutes. On the day 8, carboplatin with the calculated dose of the AUC mixed in $250 \mathrm{ml}$ of a $5 \%$ glucose solution was administered for 1 hour, following the drip infusion of gemcitabine.

\subsubsection{Paclitaxel plus Carboplatin Regimen (PC Group)}

Paclitaxel (Paclitaxel, Bristol-Myers K.K., Tokyo, Japan) was administered at of $70 \mathrm{mg} / \mathrm{m}^{2}$ on days 1,8 and 15 , and carboplatin (Paraplatin, Bristol-Myers K.K., Tokyo, Japan) with the target dose of AUC of 5 on day 1 every 28 days. As a premedication, $50 \mathrm{mg}$ of diphenhydramine hydrochloride was intravenously infused. The drip infusion of $50 \mathrm{ml}$ of an isotonic sodium chloride solution containing $8 \mathrm{mg}$ of dexamethasone sodium phosphate, 50 $\mathrm{mg}$ of ranitidine hydrochloride and $10 \mathrm{mg}$ of azasetron hydrochloride were performed. After the administration of $70 \mathrm{mg} / \mathrm{m}^{2}$ of paclitaxel in $250 \mathrm{ml}$ of a $5 \%$ glucose solution for over 1 hour, carboplatin with the calculated dose of the AUC mixed in $250 \mathrm{ml}$ of a $5 \%$ glucose solution was intravenously infused for 1 hour.

Each treatment was recommended for four cycles as a standard course. Patients could plan their scheduled cycle number between 1 to 6 and also refuse the treatment unless they met the criteria for experienced unacceptable toxicity. Full supportive therapy, corticosteroids, anticonvulsants and antibiotics were given as needed. No routine use of heatopoietic growth factors was planned. No prophylactic antibiotics were used. All patients were treated on an inpatient basis.

\subsubsection{The Exclusion Criteria}

The exclusion criteria included serious infection, fever $\left(\geq 38^{\circ} \mathrm{C}\right.$ ), impairments of the organ function (bone marrow, central nervous and cardiovascular system, liver, kidneys, interstitial pneumonia, DIC), patient's refusal 
and attending doctor's decision.

\subsection{Dose Modification}

Dose adjustments during the treatment were based on the judgement of the respective physicians-in-charge, but, as a rule, when Grade 3 or more severe nonhematotoxicity or Grade 4 hematotoxicity appeared, the doses of the anticancer drugs were reduced. The complete blood count and biochemistry were usually examined at least once or twice per week.

On the scheduled day-1 treatment, if the total leukocyte count and absolute neutrophil count (ANC) was $\leq 3.0 \times 10^{9} / 1$ and $\leq 1.5 \times 10^{9} / 1$ and/or platelet count $\leq 100 \times$ $10^{9} / 1$, respectively, the chemotherapy doses were either delayed (for up to 2 weeks) or reduced by $20 \%-25 \%$ (gemcitabine: from $1,000 \mathrm{mg} / \mathrm{m}^{2}$ to $800 \mathrm{mg} / \mathrm{m}^{2}$, AUC of carboplatin from 4 to 3; paclitaxel: from $70 \mathrm{mg} / \mathrm{m}^{2}$ to 60 $\mathrm{mg} / \mathrm{m}^{2}$, AUC of carboplatin from 5 to 4 ) to allow recovery from hematological toxicity.

On the scheduled day- 8 treatment, if the total leukocyte count and ANC was $\leq 1.0 \times 10^{9} / 1$ and $\leq 0.5 \times 10^{9} / 1$ and/or platelet count $\leq 75 \times 10^{9} / 1$, and if these parameter did not improve sufficiently, then the day- 8 administrations of drugs were postponed and omitted. A 2-week delay in initiating the subsequent course was allowed. Patients, who cannot recover from the hematological toxicity $\left(\right.$ ANC $\geq 1.0 \times 10^{9} / 1$ and/or platelet count $\left.\geq 75 \times 10^{9} / 1\right)$ within 2 weeks were withdrawn from the treatment. For patients of the delayed day- 8 and/or of the no recovery within 2 weeks for any $\geq$ grade 2 impairments of the organ function (hepatic, renal, cardiovascular, pulmonary, nervous system) and/or $\geq$ grade 3 nonhematological toxicities (excluding nausea, vomiting, anorexia, fatigue, and alopecia), the doses of the next cycle were reduced by $20 \%-25 \%$.

If these toxicities persisted after 6 weeks from day- 1 of the previous cycle, then the treatment regimen was discontinued.

The next cycle was discontinued in the case of $\geq$ Grade 3 impairments on the organ function (hepatic, renal, cardiovascular, pulmonary, nervous system) and/or $\geq$ grade 4 nonhematological toxicities (nausea, vomiting, anorexia, fatigue, and alopecia). The subsequent course of chemotherapy was begun if patients had a total leukocyte count at $\geq 3.0 \times 10^{9} / 1$, ANC $\geq 1.5 \times 10^{9} / 1$, platelet count $\geq 100 \times$ $10^{9} / 1$, creatinine $\geq 1.5 \mathrm{mg} / \mathrm{dl}$, alanine aminotransferase and aspartate aminotransferase (AST/ALT) levels $\leq 2.5$ times the normal upper limit, and total bilirubin $\leq 1.5$ times the normal upper limit, respectively. Both regimens were repeated every 4 weeks.

Regarding the use of the granulocyte colony-stimulating factor (G-CSF), patients were not to receive prophylactic G-CSF during any cycle. The use of G-CSF (100 $\mu \mathrm{g} /$ day, subcutaneous injection) was allowed only for patients who had the total leukocyte count $\leq 1.0 \times 10^{9} / \mathrm{l}$, ANC $\leq 0.5 \times 10^{9} / 1$, neutropenic fever, or documented infections with febrile neutropenia.

\subsection{Toxicity and Treatment Evaluation}

Prior to the adjuvant chemotherapy after surgery, all patients provided a complete medical history and underwent a physical examination. Patients were monitored weekly throughout treatment by physical examination, recording of toxic effects, complete blood cell counts, and blood chemistry. These patients were examined for the patient background characteristics, adverse events, treatment compliance and relapse-free survival. Adverse events were evaluated until 4 weeks after the completion of the chemotherapy according to the Common Terminology Criteria for Adverse Events Version. 3.0. The recurrence-free survival (RFS) was measured from the date of the first administration of surgery to the date of recognition of the local recurrence or distant metastasis. Overall survival (OS) was measured from the date of the first administration of surgery to the date of death or the last follow-up. The Kaplan-Meier technique [14] was used for the RFS and OS analysis. The accrual period and period of follow-up after accrual closure were 6 years and 4 months, respectively.

\subsection{Statistical Analysis}

Statistical differences in the toxicities between the two regimens were calculated using the Chi-square $\left(\chi^{2}\right)$ test. A $p$ value less than 0.05 was considered to be statistically significant.

\section{Results}

\subsection{Patient Characteristics.}

A total of 44 patients (29 patients in GC regimen and 15 in PC regimen) were completely resected in our department and summarized in Table 1. The majority of patients were males $(31 / 44,70.5 \%)$, with a median age of 64.0 years (range $45-78$ ) in the GC arm and 61.2 years (range 35 - 77) in the PC arm. The Eastern Cooperative Oncology Group Performance Status was 0 in 41 patients $(41 / 44,93.3 \%)$ and 1 in 3 patients $(3 / 44,6.8 \%)$. A lobectomy was performed in 41 patients $(41 / 44,93.2 \%)$ and a pneumonectomy was done in 3 patients $(3 / 44$, $6.8 \%$ ). Tumor histology included 39 patients with adenocarcinoma $(88.6 \%), 3$ patients with squamous cell carcinoma $(6.8 \%)$ and 2 patients with adenosquamous cell carcinoma (4.5\%). There were 2 patient with stage IB (4.5\%), 9 patients with stage IIA (20.5\%), 5 patients with stage IIB (11.4\%), 22 patients with stage IIIA (50.0\%), 3 
patients with stage IIIB $(6.8 \%)$, and 3 patients with stage IV disease $(6.8 \%)$.

\subsection{Treatment Administration}

A total of 130 chemotherapy cycles were administered (89 cycles in the GC regimen: median, 3.1; range, 2 - 4, and 41 cycles in the $\mathrm{PC}$ regimen: median, 2.7; range, 1 6) as listed in Table 2. On compliance, forty-three of 44 patients $(97.7 \%)$ completed the planned cycles, the remaining 1 patient did not complete the course due to grade 4 pneumonia. Two patients $(4.5 \%)$ received a dose reduction in the next course, and 3 patients $(6.8 \%)$ delayed the course.

\subsection{Toxicity}

For the grade 3/4 toxicities (Table 2), the PC regimen had 10 patients $(10 / 15,66.7 \%)$ and showed a higher incidence compared to twelve patients of the GC regimen (12/29, 41.4\%) in Table 2. Overall, twenty-two patients in both regimens showed grade $3 / 4$ toxicities (22/44, $50.0 \%$ ). The hematological toxicities are shown in Table 3. The grade $3 / 4$ neutropenia was significantly observed in the PC regimen (GC regimen: 12/29, 41.4\%; PC regimen: $11 / 15,73.3 \%, p=0.0443)$. The grade $3 / 4$ thrombocytopenia was more frequently seen in 6 patients (20.7\%) of the GC regimen compared to 1 patient $(6.7 \%)$ of the PC regimen $(p=0.2280)$. The grade $3 / 4$ febrile neutropenia was seen in 2 patients $(6.9 \%)$ of the GC regimen compared to 0 patient $(0 \%)$ of the $\mathrm{PC}$ regimen $(p$ $=0.2979$ ).

The nonhematological toxicities are mild except for infection as shown in Table 4. However, grade 4 pneumonia was more frequently observed in 1 patient $(1 / 15$, $6.7 \%$ ) of the PC regimen compared to none in the GC regimen $(p=0.1596)$. Grade $1 / 2$ anorexia was observed in the GC arm (GC arm: 18/29, 62.1\%; PC arm: 13/15, $86.7 \%, p=0.0900)$. Grade $1 / 2$ alanine aminotransferase and aspartate aminotransferase (ALT/AST) in the GC regimen were more significantly observed compared to those of the PC arm (GC arm: 20/29, 69.0\%; PC arm: $4 / 15,26.7 \%, p=0.0076)$. Grade $1 / 2$ alopecia was significantly observed in PC arm (GC arm: 0/25, 0.0\%; PC arm: $13 / 15,86.7 \%, p<0.0001)$. For the other toxicities, they were observed in 3 patients with headache were observed in the GC regimen. In the PC regimen, grade 4 pneumonia, headache, and hematuria in 1 patient. The overall grade $1 / 2$ non-hematologic toxicities were mild. There was no treatment-related death.

\subsection{Survival}

A survival analysis was performed in April 2011. Ten patients had been followed for less than 1 year. The median survival time (MST) of the entire GC regimen group was 784 days; 1 -year overall survival (OS) was 89.7\%, 2-year OS was $79.3 \%$, and 3 -year OS was $75.9 \%$, respectively (Table 5). The median recurrence-free survival (RFS) at 1-year, 2-year, and 3-year, was 65.5\%. The MST of the entire PC regimen group was 963 days; 1-year OS was $93.3 \%$, 2-year OS was $86.7 \%$, and 3 -year OS was $80.0 \%$, respectively. The median recurrence-free survival (RFS) at 1-year, 2-year, and 3-year, was $80.0 \%, 66.7 \%$ and $60.0 \%$, respectively.

\section{Discussion}

Five-year survival rate after surgical treatment in the United States and Japan in each pathological stage of NSCLC was reported as follows: IA, 67\%; IB, 27\%; IIA, $55 \%$; IIB, 39\%; IIIA, 38\%; IIIB, 3\% - 7\%; IV, $1 \%$ in the United States, and IA, 79.5\%; IB, 60.1\%; IIA, 59.9\%; IIB, 42.2\%; IIIA, 29.8\%; IIIB, 19.3\%; IV, 20.0\% in Japan $[2,3]$. However, up to $60 \%$ of patients with IB to IIIA NSCLC relapse after surgery and die. [2,3].

Meta-analysis of the randomized trials of adjuvant therapy of NSCLC in 1995 suggested the survival benefit of cisplatin-based chemotherapy after surgery [4]. From the meta-analysis using 9387 patients (7151 deaths) from 52 randomized clinical trials, trials comparing surgery with surgery plus chemotherapy gave a hazard ratio of

Table 3. Summary of hematological toxicities.

\begin{tabular}{|c|c|c|c|c|c|c|c|c|c|c|c|}
\hline \multirow[b]{3}{*}{ Toxicity } & \multicolumn{5}{|c|}{ GEM + CBDCA group $(n=29)$} & \multicolumn{5}{|c|}{ PTX + CBDCA group $(n=15)$} & \multirow{3}{*}{$\begin{array}{c}\mathrm{G} 3 / 4 \\
p \text { value }\end{array}$} \\
\hline & \multicolumn{5}{|c|}{$\mathrm{n}$ (\% of patients) } & \multicolumn{5}{|c|}{$\mathrm{n}$ (\% of patients) } & \\
\hline & G1 & $\mathrm{G} 2$ & G3 & G4 & $\mathrm{G} 3 / 4(\%)$ & G1 & G2 & G3 & G4 & $\mathrm{G} 3 / 4(\%)$ & \\
\hline Leukopenia & 18 & 4 & 3 & 0 & 10.3 & 5 & 7 & 1 & 0 & 6.7 & 0.6875 \\
\hline Neutropenia & 8 & 7 & 6 & 6 & 41.4 & 1 & 1 & 7 & 4 & 73.3 & 0.0443 \\
\hline Anemia & 2 & 8 & 1 & 0 & 3.4 & 4 & 0 & 1 & 0 & 6.7 & 0.6271 \\
\hline Thrombocytopenia & 2 & 7 & 6 & 0 & 20.7 & 2 & 0 & 0 & 1 & 6.7 & 0.228 \\
\hline Febrile neutropenia & 4 & 0 & 2 & 0 & 6.9 & 0 & 0 & 0 & 0 & 0 & 0.2979 \\
\hline
\end{tabular}


Table 4. Summary of nonhematological toxicities.

\begin{tabular}{|c|c|c|c|c|c|c|c|c|c|c|}
\hline \multirow[b]{3}{*}{ Toxicity } & \multicolumn{4}{|c|}{ GEM + CBDCA group $(n=29)$} & \multicolumn{4}{|c|}{ PTX + CBDCA group $(n=15)$} & \multirow{3}{*}{$\begin{array}{c}\mathrm{G} 1 / 2 \\
p \text { value }\end{array}$} & \multirow{3}{*}{$\begin{array}{c}\mathrm{G} 3 / 4 \\
p \text { value }\end{array}$} \\
\hline & \multirow[b]{2}{*}{$\mathrm{G} 1 / 2$} & \multicolumn{2}{|c|}{$\mathrm{n}$ (\% of patients) } & \multirow[b]{2}{*}{ G3/4 (\%) } & \multicolumn{4}{|c|}{$\mathrm{n}(\%$ of patients) } & & \\
\hline & & $\mathrm{G} 3 / 4$ & G1/2 (\%) & & $\mathrm{G} 1 / 2$ & $\mathrm{G} 3 / 4$ & G1/2 (\%) & G3/4(\%) & & \\
\hline Nausea & 16 & 0 & 55.2 & 0 & 7 & 0 & 46.7 & 0 & 0.5923 & - \\
\hline Vomiting & 2 & 0 & 6.9 & 0 & 2 & 0 & 13.3 & 0 & 0.4814 & - \\
\hline Anorexia & 18 & 0 & 62.1 & 0 & 13 & 0 & 86.7 & 0 & 0.09 & - \\
\hline Fatigue & 20 & 0 & 69 & 0 & 13 & 0 & 86.7 & 0 & 0.1987 & - \\
\hline Diarrhea & 3 & 0 & 10.3 & 0 & 1 & 0 & 6.7 & 0 & 0.6875 & - \\
\hline Constipation & 12 & 0 & 41.4 & 0 & 5 & 0 & 33.3 & 0 & 0.6034 & - \\
\hline ALT/AST & 20 & 0 & 69 & 0 & 4 & 0 & 26.7 & 0 & 0.0076 & - \\
\hline Creatinine & 2 & 0 & 6.9 & 0 & 1 & 0 & 6.7 & 0 & 0.6271 & - \\
\hline Neuropathy & 0 & 0 & 0 & 0 & 0 & 0 & 0 & 0 & - & - \\
\hline Pain, joint & 2 & 0 & 6.9 & 0 & 1 & 0 & 6.7 & 0 & 0.6271 & - \\
\hline Pain, muscle & 1 & 0 & 3.4 & 0 & 1 & 0 & 6.7 & 0 & 0.6271 & - \\
\hline Skin rash & 2 & 0 & 6.9 & 0 & 3 & 0 & 20 & 0 & 0.1942 & - \\
\hline Alopecia & 0 & 0 & 0 & 0 & 13 & 0 & 86.7 & 0 & $<0.0001$ & - \\
\hline Infection & 0 & 0 & 0 & 0 & $1^{* *}$ & $1^{*}$ & 6.7 & 6.7 & 0.1596 & 0.1596 \\
\hline Fever & 4 & 0 & 13.8 & 0 & 1 & 0 & 6.7 & 0 & 0.4802 & - \\
\hline \multirow[t]{2}{*}{ Others (hearing) } & 1 & 0 & 3.4 & 0 & 0 & 0 & 0 & 0 & - & - \\
\hline & \multicolumn{4}{|c|}{ GC group: headache $(n=3)$} & \multicolumn{6}{|c|}{ PC group: headache $(\mathrm{n}=1)$, hematuria $(\mathrm{n}=1)$} \\
\hline
\end{tabular}

ALT/AST: alanine aminotransferase and aspartate aminotransferase; ${ }^{*} \mathrm{G} 4$ pneumonia, ${ }^{* *} \mathrm{G} 1$ prostatitis.

Table 5. Recurrence-free and overall survivals.

\begin{tabular}{cccc}
\hline & & GEM + CBDCA group ( $\mathrm{n}=29)$ & PTX + CBDCA group $(\mathrm{n}=15)$ \\
\hline Median survival time (days) & & 784 & 963 \\
& 1-year & 65.5 & 80 \\
Recurrence-free survival (\%) & 2-year & 65.5 & 66.7 \\
& 3-year & 65.5 & 60 \\
& 1-year & 89.7 & 83.3 \\
Overall survival (\%) & 2-year & 79.3 & 86.7 \\
& 3-year & 75.9 & 80 \\
\hline
\end{tabular}

0.87 (13\% reduction in the risk of death, equivalent to an absolute benefit of 5\% at five years). However, there are no statistical differences between the postoperative adjuvant group and surgery alone [4], and this includes a number of small trials and trials with the following disability criteria and chemotherapy regimens.

After the above-mentioned meta-analysis by the Nonsmall Cell Lung Cancer Collaborative Group, several cli- nical trials examining the adjuvant chemotherapy were performed, but the efficacy of adjuvant chemotherapy remained a matter of controversy. However, useful evidence was reported after 2003. The International Adjuvant Lung Cancer Collaborative Group Trial (IALT) [15] demonstrated a $4.1 \%$ improvement in survival for patients with stage I to III NSCLC. The JBR.10 trial [16] demonstrated a $15 \%$ improvement in the 5-year survival 
for the adjuvant chemotherapy arm in stage IB or II patients. The Adjuvant Navelbine International Trialist Association (ANITA) trial [17] reported that the overall survival at 5 years improved by $8.6 \%$ in the chemotherapy arm and that this survival rate was maintained at 7 years (8.4\%) in stage II and IIIA patients. A meta-analysis based on collected and pooled individual patient data from the 5 largest randomized trials was conducted by the Lung Adjuvant Cisplatin Evaluation (LACE) [18]. This analysis demonstrated that cisplatin-based adjuvant chemotherapy improved survival in patients with stage II or III cancer. Alternatively, uracil-tegafur has been developed and tested in Japan. The Japan Lung Cancer Research Group (JLCRG) [19] on Postsurgical Adjuvant Chemotherapy reported a 5-year overall survival advantage of $11 \%$ in the uracil-tegafur (UFT) group patients with stage IB cancer. The benefit of adjuvant chemotherapy for resected stage IB, II, or IIIA NSCLC has been shown based on the results of phase III trials, such as the IALT, JBR.10, ANITA and JLCRG studies. In clinical practice, however, the 5-year survival benefit was only $5 \%$ to $10 \%$, and severe adverse reactions were seen in patients receiving cisplatin.

In 2010, the NSCLC Meta-analysis Collaborative Group [20] showed a benefit of adjuvant chemotherapy after surgery by the results that the meta-analysis of surgery plus chemotherapy versus surgery alone was based on 34 trial comparisons and 8447 patients (3323 deaths). NSCLC Meta-analyses Collaborative Group [20] recorded a benefit of adding chemotherapy after surgery (hazard ratio [HR] $0.86,95 \%$ CI $0.81-0.92, p<0.0001)$ with an absolute increase in survival of $4 \%(95 \% \mathrm{CI} 3-6)$ at 5 years (from $60 \%$ to $64 \%$ ).

There were phase III trials using four cycles of vi- norelbine and cisplatin, namely the JBR.10 [16] and ANITA trials [17]. In the JBR.10 trial, $77 \%$ of patients required at least a one-dose reduction [16]. In the ANITA trial, the median percentage of the planned doses of vinorelbine and cisplatin were $56.3 \%$ and $76.1 \%$, respectively, because of adverse events [17]. Treatment compliance has thus to date been low in the platinum-based adjuvant chemotherapy in oversea postsurgical resection patients. In the Japanese patients, the UFT trial has only been phase III trial to demonstrate survival benefits for patients whose NSCLCs were completely resected.

It is not clear whether platinum-based chemotherapy is feasible or available in an adjuvant setting for the Japanese population. A four-arm cooperative study (FACS) including the doublet of paclitaxel plus carboplatin, gemcitabine plus cisplatin, vinorelbine plus cisplatin, and irinotecan plus cisplain for Japanese patients with advanced NSCLC has been reported [21]. Based on the above results, the four regimens have similar efficacy and different toxicity profiles, and they can be used to treat advanced NSCLC patients.

The evidence report on the carboplatin plus gemcitabine regimen in Japan was few compared to those of carboplatin plus paclitaxel, almost all being reported from overseas $[22,23]$. The gemcitabine plus carboplatin regimen was allowed to administer in an outpatients setting due to the short time of drip infusion and mild nonhematological toxicities such as alopecia and gastroenterological symptoms, however, thrombocytopenia should require attention. On the other hand, carboplatin plus paclitaxel was one of the standard treatments based on much evidence reported around the world, and was appropriate to administer in an outpatients setting due to the mild toxicities. However, during the long-term administration, its neuropathy was frequently recognized [24]. For these reasons and the inconvenience of prolonged infusion, weekly administration of paclitaxel was evaluated in various cancer patients, yielding a beneficial activity and reduced toxicity [25].

The above two regimens are frequently selected for inoperable advanced lung cancer in clinical practice. In our adjuvant chemotherapy setting for the resected nonsmall cell lung cancer, on the grade $3 / 4$ toxicities, the PC regimen showed a higher incidence compared to the GC regimen. Grade 3/4 leukopenia was significantly observed in the PC regimen, and grade 3/4 thrombocytopenia was more frequently seen in the $\mathrm{GC}$ regimen compared to those in the PC regimen. Grade 3/4 febrile neutropenia was seen in the $\mathrm{GC}$ regimen compared to none in the $\mathrm{PC}$ regimen. The nonhematological toxicities were mild. However, grade 4 pneumonia was more frequently observed in the PC regimen compared to none in the GC regimen.

Based on the results of the above-mentioned our clinical outcomes, the toxicities of the GEM + CBDCA and PTX + CBDCA groups for the resected non-small lung cancer were different, however, as adjuvant chemotherapy, both regimens were feasible and safely performed in the postoperative period. The adjuvant GEM + CBDCA and PTX + CBDCA treatments were well tolerated, and there were no chemotherapy-related deaths. Accordingly, the GEM + CBDCA and PTX+CBDCA regimens could be considered as a treatment option for Japanese surgical patients.

\section{REFERENCES}

[1] A. Jemal, T. Murray, E. Ward, A. Samuels, R. C. Tiwari, A. Ghafoor, E. J. Feuer and M. J. Thun, "Cancer Statistics, 2005," CA: A Cancer Journal for Clinicians, Vol. 55, No. 1, 2005, pp. 10-30. doi:10.3322/canjclin.55.1.10

[2] C. F. Mountain, "Revisions in the International System for Staging Lung Cancer," Chest, Vol. 111, No. 6, 1997, 
pp. 1710-1717. doi:10.1378/chest.111.6.1710

[3] T. Goya, H. Asamura, H. Yoshimur, H. Kato, K. Shimokata, R. Tsuchiya, Y. Sohara, T. Miya and E. Miyaoka (Japanese Joint Committee of Lung Cancer Registry), "Prognosis of 6644 Resected Non-Small Cell Lung Cancers in Japan: A Japanese Lung Cancer Registry Study," Lung Cancer, Vol. 50, No. 2, 2005, pp. 227-234. doi:10.1016/j.lungcan.2005.05.021

[4] Non-Small Cell Lung Cancer Collaborative Group, "Chemotherapy in Non-Small Cell Lung Cancer: A MetaAnalysis Using Updated Data on Individual Patients from 52 Randomised Clinical Trials," BMJ, Vol. 311, No. 7010, 1995, pp. 899-909. doi:10.1136/bmj.311.7010.899

[5] S. M. Keller, S. Adak, H. Wagner, A. Herskovic, R. Komaki, B. J. Brooks, M. C. Perry, R. B. Livingston and D. H. Johnson, "A Randomized Trial of Postoperative Adjuvant Therapy in Patients with Completely Resected Stage II or IIIA Non-Small-Cell Lung Cancer. Eastern Cooperative Oncology Group," The New England Journal of Medicine, Vol. 343, No. 17, 2000, pp. 1217-1222. doi:10.1056/NEJM200010263431703

[6] Y. Ichinose, H. Tada, T. Koike, N. Nishizawa, R. Tsuchiya, K. Nagai, et al., "A Randomized Phase III Trial of Postoperative Adjuvant Chemotherapy in Patients with Completely Resected Stage IIIA-N2 Non-Small Cell Lung Cancer: Japan Clinical Oncology Group (JCOG) 9304 Trial," Proceeding of ASCO 2001, Vol. 20, 2001, Article ID: A1241.

[7] G. V. Scagliotti, R. Fossati, V. Torri, L. Crinò, G. Giaccone, G. Silvano, M. Martelli, M. Clerici, F. Cognetti and M. Tonato (Adjuvant Lung Project Italy/European Organisation for Research Treatment of Cancer-Lung Cancer Cooperative Group Investigators), "Randomized Study of Adjuvant Chemotherapy for Completely Resected Stage I, II, or IIIA Non-Small-Cell Lung Cancer," Journal of the National Cancer Institute, Vol. 95, No. 19, 2003, pp. 1453-1461. doi:10.1093/jnci/djg059

[8] D. Waller, D. J. Fairlamb, N. Gower, R. Milroy, M. D. Peake, R. M. Rudd, et al., "The Big Lung Trial (BLT): Determining the Value of Cisplatin-Based Chemotherapy for All Patients with Non-Small Cell Lung Cancer (NSCLC). Preliminary Results in the Surgical Setting," Proceeding of ASCO 2003, Vol. 22, 2003, Article ID: A2543.

[9] R. Arriagada, B. Bergman, A. Dunant, T. Le Chevalier, J. P. Pignon and J. Vansteenkiste (International Adjuvant Lung Cancer Trial Collaborative Group), "CisplatinBased Chemotherapy in Patients with Completely Resected Non-Small Cell Lung Cancer," The New England Journal of Medicine, Vol. 350, No. 2, 2004, pp. 351-360.

[10] L. H. Sobin and I. D. Fleming, "TNM Classification of Malignant Tumors, Fifth Edition (1997). Union Internationale Contre le Cancer and the American Joint Committee on Cancer," Cancer, Vol. 80, No. 9, 1997, pp. 1803-1804.

[11] W. D. Travis, K. Garg, W. A. Franklin, I. I. Wistuba, B. Sabloff, M. Noguchi, R. Kakinuma, M. Zakowski, M. Ginsberg, R. Padera, et al., "Bronchioloalveolar Carcinoma and Lung Adenocarcinoma: The Clinical Impor- tance and Research Relevance of the 2004 World Health Organization Pathologic Criteria," Journal of Thoracic Oncology, Vol. 1, No. 9, 2006, pp. S13-S19. doi:10.1097/01243894-200611001-00004

[12] A. H. Calvert, D. R. Newell, L. A. Gumbrell, S. O'Reilly, M. Burnell, F. E. Boxall, Z. H. Siddik, I. R. Judson, M. E. Gore and E. Wiltshaw, "Carboplatin Dosage: Prospective Evaluation of a Simple Formula Based on Renal Function," Journal of Clinical Oncology, Vol. 7, No. 11, 1989, pp. $1748-1756$.

[13] D. W. Cockcroft and M. H. Gault, "Prediction of Creatinine Clearance from Serum Creatinine," Nephron, Vol. 16, No. 1, 1976, pp. 31-41.

[14] E. L. Kaplan and P. Meier, "Non-Parametric Estimation from Incomplete Observations," Journal of the American Statistical Association, Vol. 53, 1958, pp. 457-481. doi:10.1080/01621459.1958.10501452

[15] R. Arriagada, B. Bergman, A. Dunant, T. Le Chevalier, J. P. Pignon and J. Vansteenkiste (International Adjuvant Lung Cancer Trial Collaborative Group), "Cisplatin-Based Adjuvant Chemotherapy in Patients with Completely Resected Non-Small-Cell Lung Cancer," The New England Journal of Medicine, Vol. 350, No. 4, 2004, pp. 351-360. doi:10.1056/NEJMoa031644

[16] T. Winton, R. Livingston, D. Johnson, J. Rigas, M. Johnston, C. Butts, Y. Cormier, G. Goss, R. Inculet, E. Vallieres, et al., "National Cancer Institute of Canada Clinical Trials Group; National Cancer Institute of the United States Intergroup JBR.10 Trial Investigators. Vinorelbine plus Cisplatin vs. Observation in Resected NonSmall-Cell Lung Cancer," The New England Journal of Medicine, Vol. 352, No. 25, 2005, pp. 2589-2597. doi:10.1056/NEJMoa043623

[17] J. Y. Douillard, R. Rosell, M. De Lena, F. Carpagnano, R. Ramlau, J. L. Gonzáles-Larriba, T. Grodzki, J. R. Pereira, A. Le Groumellec, V. Lorusso, et al., "Adjuvant Vinorelbine plus Cisplatin versus Observation in Patients with Completely Resected Stage IB-IIIA Non-Small-Cell Lung Cancer (Adjuvant Navelbine International Trialist Association [ANITA]): A Randomised Controlled Trial," Lancet Oncology, Vol. 7, No. 9, 2006, pp. 719-727. doi:10.1016/S1470-2045(06)70804-X

[18] J. P. Pignon, H. Tribodet, G. V. Scagliotti, J. Y. Douillard, F. A. Shepherd, R. J. Stephens, A. Dunant, V. Torri, R. Rosell, L. Seymour, et al., "LACE Collaborative Group. Lung Adjuvant Cisplatin Evaluation: A Pooled Analysis by the LACE Collaborative Group," Journal of Clinical Oncology, Vol. 26, No. 21, 2008, pp. 3552-3559. doi:10.1200/JCO.2007.13.9030

[19] H. Kato, Y. Ichinose, M. Ohta, E. Hata, N. Tsubota, H. Tada, Y. Watanabe, H. Wada, M. Tsuboi, N. Hamajima, et al., "Japan Lung Cancer Research Group on Postsurgical Adjuvant Chemotherapy. A Randomized Trial of Adjuvant Chemotherapy with Uracil-Tegafur for Adenocarcinoma of the Lung," The New England Journal of Medicine, Vol. 350, No. 17, 2004, pp. 1713-1721. doi:10.1056/NEJMoa032792

[20] NSCLC Meta-Analyses Collaborative Group, R. Arriagada, A. Auperin, S. Burdett, J. P. Higgins, D. H. Johnson, 
T. Le Chevalier, C. Le Pechoux, M. K. Parmar, J. P. Pignon, R. L. Souhami, et al., "Adjuvant Chemotherapy, with or without Postoperative Radiotherapy, in Operable Non-Small-Cell Lung Cancer: Two Meta-Analyses of Individual Patient Data," Lancet, Vol. 375, No. 9722, 2010, pp. 1267-1277. doi:10.1016/S0140-6736(10)60059-1

[21] Y. Ohe, Y. Ohashi, K. Kubota, T. Tamura, K. Nakagawa, S. Negoro, Y. Nishiwaki, N. Saijo, Y. Ariyoshi and M. Fukuoka, "Randomized Phase III Study of Cisplatin plus Irinotecan versus Carboplatin plus Paclitaxel, Cisplatin plus Gemcitabine, and Cisplatin plus Vinorelbine for Advanced Non-Small-Cell Lung Cancer: Four-Arm Cooperative Study in Japan," Annals of Oncology, Vol. 18, No. 2, 2007, pp. 317-323. doi:10.1093/annonc/mdl377

[22] P. Zatloukal, L. Petruzelka, M. Zemanová, V. Kolek, J. Skricková, M. Pesek, H. Fojtů, I. Grygárková, D. Sixtová, J. Roubec, et al., "Gemcitabine plus Cisplatin vs. Gemcitabine plus Carboplatin in Stage IIIb and IV Non-Small Cell Lung Cancer: A Phase III Randomized Trial," Lung Cancer, Vol. 41, No. 3, 2003, pp. 321-331. doi:10.1016/S0169-5002(03)00233-2

[23] P. Rocchi, C. Mattioli,. M. B. Rocchi, R. Mattioli, P.
Lippe, R. Trivisonne, F. Buzzi, G. De Signoribus, G. Tuveri, G. Rossi, et al., "Randomized, Multicenter, Phase II Study of Gemcitabine plus Cisplatin versus Gemcitabine plus Carboplatin in Patients with Advanced Non-Small Cell Lung Cancer," Lung Cancer, Vol. 41, No. 1, 2003, pp. 81-89. doi:10.1016/S0169-5002(03)00140-5

[24] K. Kelly, J. Crowley, P. A. Bunn Jr., C. A. Presant, P. K., Grevstad, C. M. Moinpour, S. D. Ramsey, A. J. Wozniak, G. R. Weiss, D. F. Moore, et al., "Randomized Phase III Trial of Paclitaxel plus Carboplatin versus Vinorelbine plus Cisplatin in the Treatment of Patients with Advanced Non-Small-Cell Lung Cancer: A Southwest Oncology Group Trial," Journal of Clinical Oncology, Vol. 19, No. 13, 2001, pp. 3210-3218

[25] M. Hirabayashi, K. Endoh, M. Teramachi, M. Okuda, K. Yamaguchi, K. Fukuda, H. Tokuhisa, H. Kagioka, N. Nakai and M. Nakade, "Phase II Study of Carboplatin and Weekly Paclitaxel Combination Chemotherapy in Advanced Non-Small Cell Lung Cancer: A Kansai Clinical Oncology Group Study," Lung Cancer, Vol. 44, No. 3, 2004, pp. 355-362. doi:10.1016/j.lungcan.2003.12.001 